\title{
Homology of Simploidal Set
}

\author{
Samuel Peltier, Laurent Fuchs, and Pascal Lienhardt \\ SIC, Université de Poitiers, Boulevard Marie et Pierre Curie \\ 86962 Futuroscope Chasseneuil Cedex, France \\ \{peltier, fuchs, lienhardt\}@sic.univ-poitiers.fr
}

\begin{abstract}
In this article the homology of simploidal sets is studied. Simploidal sets generalize both simplicial complexes and cubical complexes, more precisely cells of simplicial sets are cartesian products of simplices. We define one homology for simploidal sets and we prove that this homology is equivalent to the homology usually defined on simplicial complexes.
\end{abstract}

\section{Introduction}

The aim of this paper is to define and to study the homology of simploidal sets. Simploidal sets (see Fig. 1 $(a)$ ) can be considered as a special case of cellular complexes, where cells are simploids [1,2], i.e. products of simplices. Simploidal sets include simplicial complexes and cubical complexes as particular cases, so they can be used for representing the topology of digital images. They can also be used for representing hybrid grids coming from finite elements methods. The notion of simploid was introduced by DAHMEN and MICCHELLI [1 to study multivariate splines.

Topological invariants provide information about the structure of an object. Homology is a powerful on 1 which can be computed for any dimension. Homology groups describe dimensional "holes" of a combinatorial object (connected components for dimension 0 , holes for dimension 1 , cavities for dimension $2, \ldots$ ). Homology information can be represented on combinatorial structures by computing homology groups generators. For example, Fig. 1 $(c)$ represents the two 1 -dimensional holes of the torus $(b)$.

For digital image analysis, topological invariants are useful for classification, indexation, or shape description [3]. Homology groups are classically computed for simplicial combinatorial structures such as abstract simplicial complexes [4] or semi-simplicial sets [5]. In this paper, we show that it is always possible to convert a simploidal set into a simplicial structure (a semi-simplicial set). So, homology groups of a simploidal set can be computed from the corresponding semi-simplicial set. Since many simplices correspond to a single simploid, this conversion of data structures can be space and time consuming. Similar arguments as those developed for cubical complexes [6] can be taken into account.

${ }^{1}$ Homology groups contain other classical topological invariant as Euler characteristic, Betti numbers, and orientability of a closed surface. 
We propose a direct definition of homology groups for simploidal sets, i.e. we define boundary homomorphisms from which we can construct a free chain complex 2 . It is well-known from algebraic topology that homology defined on a triangulable cell complex (in our case : a simploidal set), is equivalent to homology defined on the associated triangulated space (in our case : the associated semi-simplicial set). We study this equivalence in a combinatorial and constructive way in order to compute simplicial generators from simploidal generators and conversely.

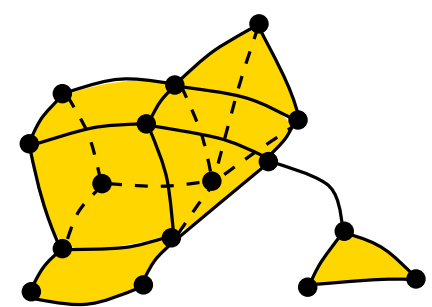

(a)

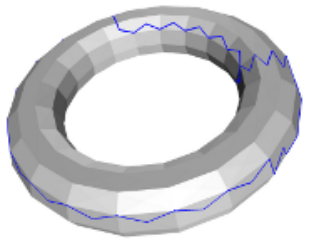

(b)

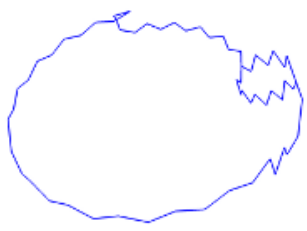

(c)

Fig. 1. (a) : a simploidal object. $(b)-(c)$ : a geometric representation of the two $1-$ holes of the torus.

In section 2 we recall the definition of semi-simplicial sets and basic concepts of chain, cycle, boundary, free chain complex and homology groups. In section 3. the simploidal set definition is recalled. Boundary homomorphisms for this structure is defined, and thus homology groups of these sets can be defined by constructing a free chain complex. In section 4 , we study the conversion of a simploidal set into a corresponding semi-simplicial set. Then, We define a morphism between simplicial and simploidal chain groups, which associates to each simploidal chain an equivalent simplicial chain in the corresponding semisimplicial set. After, we describe algorithms for constructing a simplicial homology generator from a simploidal one, and conversely. This construction provides a combinatorial and constructive proof of the equivalence between simploidal and simplicial homologies.

\section{Homology of Semi-simplicial Sets}

In this section all notions needed to define the homology groups over a combinatorial structure are introduced. Semi-simplicial sets [5,7] are used to illustrate these notions. Since our goal is the computation of homology groups of objects explicitly represented within a computer, all sets are finite.

${ }^{2}$ A free chain complex is an algebraic structure from which homology groups are defined (cf. section 2). 


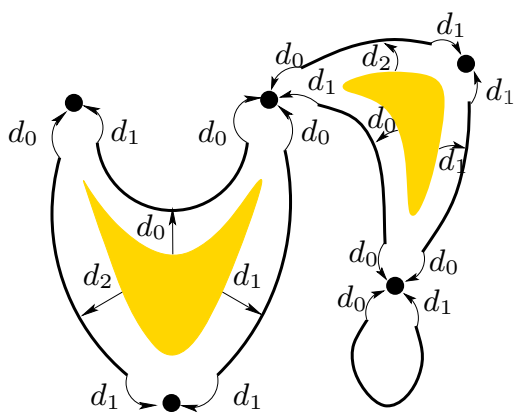

(a)

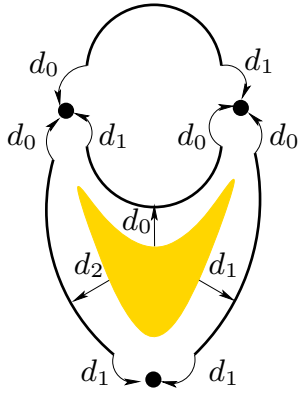

(b)

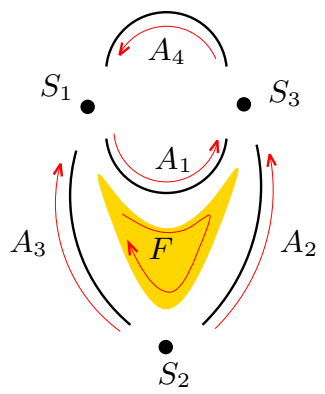

(c)

Fig. 2. $(a)-(b)$ : Examples of semi-simplicial sets. $(c)$ : positive orientation of simplices of $(b)$.

\subsection{Semi-simplicial Sets}

Definition 1. [5] Let $n \in \mathbb{N}$. A n-dimensional semi-simplicial set $S=$ $\left(K,\left(d_{i}^{p}\right)\right)$ is a family of sets $K=\left(K^{p}\right)_{p \in[0 . . n]}$ together with maps $d_{i}^{p}: K^{p} \rightarrow K^{p-1}$ for $i=0, \ldots, p$, which satisfy the following property :

$$
\forall p, 2 \leq p \leq n, \forall i, j, 0 \leq j<i \leq n, \forall \sigma \in K^{p}, \sigma d_{i}^{p} d_{j}^{p-1}=\sigma d_{j}^{p} d_{i-1}^{p-1}
$$

The elements of $K^{p}$ are $p$-simplices, the $d_{i}^{p}$ are boundary operators (the superscripts $p$ will be usually dropped). The relations between the boundary operators ensure that simplices are coherently assembled. Without these relations, a $p$-simplex could have more than $p+1$ distinct vertices in its boundary, i.e. $\sigma d_{2} d_{1}, \sigma d_{2} d_{0}, \sigma d_{1} d_{1}, \sigma d_{1} d_{0}, \sigma d_{0} d_{1}, \sigma d_{0} d_{0}$ : relations between boundary operators ensure that $\sigma d_{2} d_{1}=\sigma d_{1} d_{1}, \sigma d_{2} d_{0}=\sigma d_{0} d_{1}, \sigma d_{1} d_{0}=\sigma d_{0} d_{0}$. The notion of semisimplicial set generalizes the classical notion of abstract simplicial complexes [4] in the following way: a semi-simplicial set can be associated to any abstract simplicial complex, but the converse is not true. For example, it is not possible to associate an abstract simplicial complex with the semi-simplicial set of Fig $2(a)$, since it contains a self-loop.

\subsection{Chain, Boundary Homomorphism and Free Chain Complex}

Let $n_{p}$ be the number of $p$-simplices of $K^{p}$, and $K^{p}=\left\{\sigma_{1}^{p}, \cdots, \sigma_{n_{p}}^{p}\right\}$. A $p$-chain $c$ is a combination of $p$-simplices together with integer coefficients : $c=\sum_{i=1}^{n_{p}} \alpha_{i}^{p} \sigma_{i}^{p}$. For example on Fig. 2( $(c): A_{1},-A_{2}$ and $3 A_{2}-A_{4}$ are 1 -chains. The addition of $p$-chains consists in the addition of the corresponding simplex coefficients. The neutral element is the empty chain denoted 0 for each dimension.

${ }^{3}$ It could be noted that the notation $x f$ is used instead of the classical notation $f(x)$ as it is more convenient when handling boundary operators. 
For any $p$, the $p$-chain group $C_{p}$ is a free abelian group with $K^{p}$ as a basis [4]. The chain concept is a purely formal one, since multiplying a $p$-simplex $\sigma_{i}^{p}$ by an integer has no geometric meaning except for 1 and -1 . In these cases $1 . \sigma_{i}^{p}$ means that we consider $\sigma_{i}^{p}$ with its orientation and $-1 . \sigma_{i}^{p}$ means that we consider $\sigma_{i}^{p}$ with its opposite orientation. The orientation of each simplex is induced by its boundary operators (c.f. Fig. $2(c)$ and the following definition). More formal discussions about orientation can be found in [4].

Homology groups are defined from the sequence of chain groups and applications $\partial_{i}$ defined between these groups, $C_{n} \stackrel{\partial_{n}}{\longrightarrow} C_{n-1} \stackrel{\partial_{n-1}}{\longrightarrow} \cdots \stackrel{\partial_{1}}{\longrightarrow} C_{0} \stackrel{\partial_{0}}{\longrightarrow} 0$. These applications satisfy the relation $c^{p} \partial_{p} \partial_{p-1}=0$ for each $p$-chain $c^{p}, p \geq 1$. Such a sequence is a free chain complex. The image of $c^{p}$ by application $\partial_{p}$ is the boundary of $c^{p}$.

Definition 2. For any $p, 1 \leq p \leq n$, the boundary of $p$-simplex $\sigma^{p}$ is the $(p-1)-$ chain $\sigma^{p} \partial_{p}=\sum_{i=0}^{p}(-1)^{i} \sigma d_{i}$. The boundary of a 0 -simplex is the null chain.

The definition of a $p$-chain boundary is directly deduced by linearity from the definition of a $p$-simplex boundary and the boundary applications $\partial_{p}$ are homomorphisms. Usually the subscript and superscript $p$ will be dropped. For example on Fig. 2( $2(c): F \partial=A_{1}-A_{2}+A_{3}$ and $\left(4 A_{2}-3 A_{4}\right) \partial=4\left(A_{2}\right) \partial-3\left(A_{4}\right) \partial=$ $7 S_{3}-4 S_{2}-3 S_{1}$ are chain boundaries. Note that $F \partial \partial=\left(A_{1}-A_{2}+A_{3}\right) \partial=0$.

In order to verify that applications $\partial_{p}$ are actually boundary applications, we only have to check that $c \partial \partial=0$ for each chain $c$ composed by one simplex. This can directly be done using definition 2 and the property that for any $p-\operatorname{simplex} \sigma, \sigma \partial \partial=0$.

\subsection{Cycles, Boundaries, Homology Groups}

In order to define homology groups, we first define particular chains. A $p$-chain which boundary is null is a $p$-cycle. The set of $p$-cycles equipped with the addition is a $p$-chain subgroup, denoted $Z_{p}$. For example on Fig. $2(c)$ : 1 -chains $A_{1}-A_{2}+A_{3}$ and $A_{1}+A_{4}$ are $1-$ cycles: $\left(A_{1}-A_{2}+A_{3}\right) \partial=\left(S_{3}-S_{1}\right)-\left(S_{3}-\right.$ $\left.S_{2}\right)+\left(S_{1}-S_{2}\right)=0$ and $\left(A_{1}+A_{4}\right) \partial=\left(S_{3}-S_{1}\right)+\left(S_{1}-S_{3}\right)=0$.

A $p$-chain which is the boundary of a $(p+1)$-chain is a $p$-boundary. The set of $p$-boundaries equipped with the addition is also a $p$-chain subgroup, denoted $B_{p}$. Moreover, each $p$-boundary is a $p$-cycle (since $\forall c \in C^{p+1}, c \partial \partial=0$ ) hence $B_{p}$ is a subgroup of $Z_{p}$. For example on Fig. $2(c): 1-$ chain $A_{1}-A_{2}+A_{3}$ is the boundary of 2-chain $F$.

A $p$-dimensional hole is a $p$-cycle which is not a $p$-boundary. For example, on Fig. 2(c), 1-cycle $A_{1}+A_{4}$ is not a boundary.

Now an equivalence relation is defined as follow: two $p$-cycles $\mu_{1}$ and $\mu_{2}$ are equivalent if their difference is a boundary, i.e. $\mu_{1}=\mu_{2}+c \partial_{p+1}: \mu_{1}$ and $\mu_{2}$ are homologous 4 . Homology group $H_{p}$ is the quotient of cycle group $Z_{p}$ by the equivalence relation (i.e. $H_{p}=Z_{p} / B_{p}$ ). Hence two cycles belong to the same

\footnotetext{
${ }^{4}$ As a special case, if $\mu=c \partial_{p+1}$ then $\mu$ is homologous to 0 .
} 
equivalence class if they surround the same hole. For example on Fig. 2( $(c)$ : cycles $z_{2}=A_{2}-A_{3}+A_{4}$ and $A_{1}+A_{4}$ are homologous, since $z_{1}=z_{2}+F \partial$.

For any $p, H_{p}$ is finitely generated, i.e. there is a finite number of elements from which all others can be deduced. Hence, following the finitely generated group theorem, any group $H_{p}$ is isomorphic to a direct sum [4]:

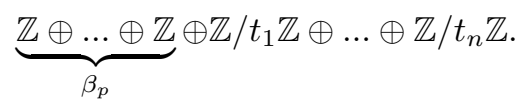

Each $\mathbb{Z}$ corresponds to an equivalence class of infinite order cycles 5 . The number $\beta_{p}$ is the $p$ th Betti number. Each $\mathbb{Z} / t_{i} \mathbb{Z}$ corresponds to an equivalence class of cycles of finite order $t$. Integers $t_{i}$ are the torsion coefficients. A cycle of finite order is a weak boundary.

\section{Homology of Simploidal Sets}

In this section, simploidal sets are introduced. We extend classical notions of chains, cycles and boundaries and we define boundary homomorphisms for this structure. Thus, we provide a direct homology definition for simploidal sets.

\subsection{Simploidal Sets}

A simploid can be defined as the product of polytopes, which are "geometric" simplices [2]. We recall here the combinatorial structure of simploidal sets, which is based upon the notion of semi-simplicial set (see section 4). In a simploidal set, a simploid is defined by a $k$-tuple $\left(a_{1}, \ldots, a_{k}\right)$ of strictly positive integers, which is its type, $k$ is the length of the simploid, $\sum_{l=1}^{k} a_{l}$ is its dimension (intuitively, a simploid is the product of simplices of respective dimensions $a_{1}, \cdots a_{k}$ ). Some examples of simploids are shown on Fig. 3. It should be noted that a $p$-simplex is a simploid of type $(p)$ and that a $p$-cube is a simploid of type $(1, \ldots, 1)$ with length $p$.

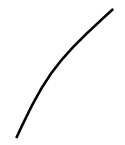

(1)

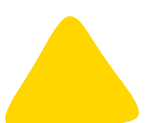

(2)

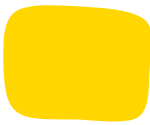

$(1,1)$

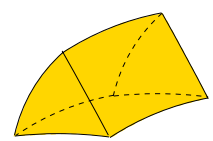

$(2,1)$

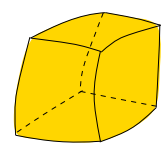

$(1,1,1)$

Fig. 3. Examples of simploids

\footnotetext{
${ }^{5}$ For any $p, h \in H_{p}$ is an infinite order cycle if and only if, for any $\alpha, \alpha h \notin B_{p}$.

${ }^{6}$ For any $p, h \in H_{p}$ is a cycle of order $t_{i}$ if and only if, for any $\alpha \in\left[1 . . t_{i}-1\right], \alpha h \notin B$ and $t_{i} h \in B$.
} 
Definition 3. [8] A simploidal set $S=\left(K,\left(\epsilon_{j}^{i}\right)\right)$ of dimension $n$ is the union $\bigcup_{p=0}^{n} K^{p}$ of sets of $p$-dimensional simploids, $0 \leq p \leq n$ equipped with border operators $\epsilon_{j}^{i}$ such that:

$$
\begin{aligned}
&\left(\ldots, a_{i}, \ldots\right) \epsilon_{j}^{i}:\left\{\begin{array}{l}
\left(\ldots, a_{i}-1, \ldots\right) \text { if } a_{i}>1 \\
\left(\ldots, \widehat{a}_{i}, \ldots\right) \quad \begin{array}{l}
\text { otherwise }\left(\widehat{a}_{i}\right. \text { means } \\
\left.a_{i} \text { is removed }\right)
\end{array}
\end{array}\right. \\
&\left(\ldots, a_{i}, \ldots\right) \epsilon_{k}^{i} \epsilon_{l}^{i}=\left(\ldots, a_{i}, \ldots\right) \epsilon_{l}^{i} \epsilon_{k-1}^{i} \quad \text { with } k>l \text { and } a_{i}>1
\end{aligned}
$$

Figure 4(a) shows an example of simploidal set. In the previous definition, first equation (11) denotes the action of a border operator on the simploid type. The cartesian product of a simploid $s$ by a simploid of type (0) (i.e. a vertex) is the identity. Hence, if zero appears in the type of a simploid by the application of a boundary operator, it is removed from the type. With equation (2), the commutation relation for semi-simplicial set boundary operators is retrieved. Finally, equation (3) is the commutation relation when two boundary operators are successively applied to two different simplices. The second part of this equation allows us to take into account the shifts that are produced by suppressed zeros.

For example, if we apply the sequence of boundary operators $\epsilon_{0}^{3} \epsilon_{1}^{2}$ to a simploid of type $(2,1,1)$, we obtain first a simploid $(2,1)$, due to the application of $\epsilon_{0}^{3}$ and after a simploid (2) by the application of $\epsilon_{1}^{2}$. In an other way, if we start by the application of $\epsilon_{1}^{2}$, a simploid $(2,1)$ is obtain since the zero that appears in the middle of the type is removed. Hence, we cannot apply operator $\epsilon_{0}^{3}$. The applied operator is $\epsilon_{0}^{2}$, so we get $(2,1,1) \epsilon_{0}^{3} \epsilon_{1}^{2}=(2,1,1) \epsilon_{1}^{2} \epsilon_{0}^{2}$.

\subsection{Simploidal Chain, Boundary Homomorphism and Free Chain Complex}

In order to define simploidal homology, we have to associate a free chain complex to a simploidal set. Let $S=\left(K,\left(\epsilon_{j}^{i}\right)\right)$ be a simploidal set: a simploidal $p$-chain is a combination of simploids of $K^{p}$ with integer coefficients. Now, to define boundary homomorphisms $\partial^{\square}$ for simploidal sets, we extend the general boundary formula of a cell-product: $(a \times b) \partial=a \partial \times b+(-1)^{\operatorname{dim}(a)} a \times b \partial$.

Definition 4. Let $s$ be a simploid of type $\left(a_{1}, \cdots, a_{k}\right)$.

$$
s \partial^{\square}= \begin{cases}0 & \text { if } s=() \\ \sum_{i=1}^{k} \sum_{j=0}^{a_{i}}(-1)^{j+\sum_{l=1}^{i-1} a_{l}} s \epsilon_{j}^{i} & \text { otherwise }\end{cases}
$$




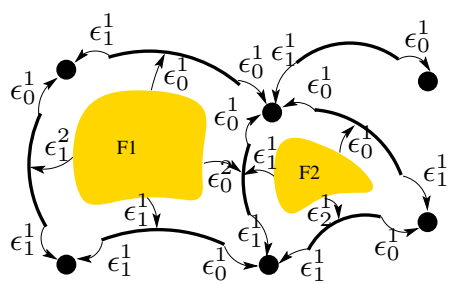

(a)

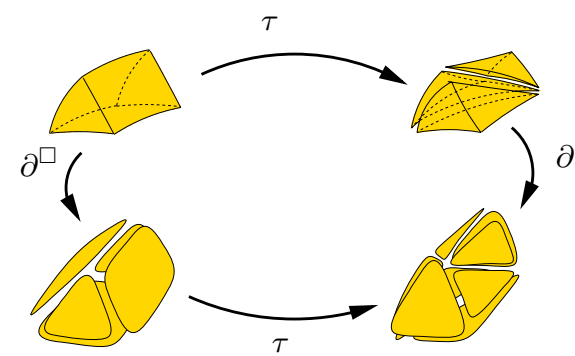

(b)

Fig. 4. (a) an example of simploidal set of dimension 2. (b) a commutative diagram which illustrates the property $c \tau \partial=c \partial^{\square} \tau$.

For example on Fig. 4(a) $F_{1} \partial^{\square}=\left(F_{1} \epsilon_{0}^{1}-F_{1} \epsilon_{1}^{1}\right)-\left(F_{1} \epsilon_{0}^{2}-F_{1} \epsilon_{1}^{2}\right) ; F_{2} \partial^{\square}=$ $F_{2} \epsilon_{0}^{1}-F_{2} \epsilon_{1}^{1}+F_{2} \epsilon_{2}^{1}$. Definition 4 is extended by linearity for simploidal chains.

To prove that $c \partial^{\square} \partial^{\square}=0$ for any simploidal chain $c$ (i.e. $\partial^{\square}$ are boundary homomorphisms), we prove this property for a simploidal chain containing a single simploid $s$ (linearity ensure extension for a general chain). Then, we prove that definition 4 satisfies the general boundary formula of a cell-product7 and conclusion follows. So, we can associate a free chain complex to a simploidal set. Now we are able to compute the homology groups for such a set, using for example the SMITH normal form transformation for incidence matrices [4.

\section{Conversion Between Simploidal and Semi-simplicial Sets}

As we will see in this section, it is always possible to associate a semi-simplicial set with a simploidal set. And, it is well known in algebraic topology that the homology of a triangulable space does not depend on its triangulation [4. So we can directly conclude that simploidal homology as defined in section 3 is equivalent to simplicial homology.

In this section, we study conversions between semi-simplicial and simploidal sets. We define operator $\mathfrak{T}$ which associates to each simploid a set of simplices in the associated semi-simplicial set. We also define operator $\tau$, which associates to each $p$-simploidal chain a $p$-simplicial chain in the associated semi-simplicial set. Operator $\tau$ preserves the boundary i.e. for any simploidal chain $c, c \tau \partial=$ $c \partial^{\square}$ (see Fig. 4(b)).

Finally, we provide algorithms for converting a simploidal chain into a simplicial chain and conversely. So we can associate a simplicial generator with each simploidal homology generator and conversely. Incidentally, we get a direct and constructive proof of the equivalence between simploidal and simplicial homology.

\footnotetext{
7 This can be directly be proved using a recursion over the length of a simploid.
} 


\subsection{Semi-simplicial Set Associated with a Simploidal Set}

Any simploidal set can be constructed in two steps: (1) Creation of principa 8 simploids and their boundaries. (2) Identifications 9 of simploids which are in the boundary of principal simploids.

In order to construct the semi-simplicial set associated to a simploidal set, we proceed as follow : (a) For each simploid, the set of corresponding simplices is created in the associated semi-simplicial set. (b) Boundary operators are defined between simplices that correspond to a same simploid $s$ and between simplices that corresponds to $s$ and $s \partial^{\square}$.

Cartesian Product of Semi-simplicial Sets; Triangulation of a Simploid. We recall some notions related to the cartesian product. The definition is not provided, since it is rather long and it will not be used here. Actually, the cartesian product operation is defined on simplicial sets, which extend semi-simplicial sets by adding a second class of operators (degeneracy operators), which induces a second type of simplices (degenerate simplices, see [5]). The general definition of simplicial sets makes possible to define cartesian product in a very simple way. From which an equivalent definition of cartesian product which acts directly upon semi-simplicial sets $[9$ is deduced. The basic principle is the following : the cartesian product of two simplices is made of simplices (maybe having different dimensions), which can be identified by integer sequences (these integer sequences correspond to sequences of degeneracy operators). Boundary operators can also be deduced from these integer sequences and relations with boundary operators of the initial simplices.

In practice, the product of $l$-simplex $\sigma$ and $m$-simplex $\mu$, such that $l \geq m$, is a set of simplices of dimensions $l$ to $l+m$, where the set of $r$-dimensional simplices $(l \leq r \leq l+m)$ corresponds to the set of simplices denoted $(\sigma I, \mu J)$, for all disjoint sequences $I$ and $J$ such that :

$-I=\left(i_{1} \cdots i_{r-l}\right), J=\left(j_{1} \cdots j_{r-m}\right)$

$-0 \leq i_{1}<\ldots<i_{r-l} \leq r-1,0 \leq j_{1}<\ldots<j_{r-m} \leq r-1$

For instance Fig. [5(c) illustrates the cartesian product of the two semi-simplicial sets $(a)$.

Then it is possible to define the set of simplices associated with a simploid of length 2. We can extend this definition for any simploid $s=\sigma_{1} \times \cdots \sigma_{n}$. The set of associated simplices is denoted $s \mathfrak{T}$. From $s \mathfrak{T}$ the set of simplices of dimension $d=a_{1}+\cdots+a_{n}$, is $s T=\left\{\left(\left(\cdots\left(\left(\sigma_{1} I_{1}, \sigma_{2} \bar{I}_{1}\right) I_{2}, \sigma_{3} \bar{I}_{2}\right) \cdots\right) I_{n-1}, \sigma_{n} \bar{I}_{n-1}\right)\right\}$, where:

$I_{i} \in E^{a_{i+1}, a_{1}+\cdots+a_{i+1}}, 1 \leq i \leq n-1$,

$E^{p, n}$ is the set of strictly increasing integer sequences of $p$ integers range 0 to $n-1$,

If $I$ is an element of $E^{p, n}$, we denote $\bar{I}$ the sequence of $E^{n-p, n}$ such that $I \cap \bar{I}=\emptyset$.

\footnotetext{
${ }^{8}$ A simploid is a main simploid if it is not in the boundary of another simploid.

${ }^{9}$ Intuitively, identifying two simploids consists in merging them.
} 
For instance, let $\sigma_{1}$ (reps. $\sigma_{2}, \sigma_{3}$ ) be a 1 -simplex (resp. 3-simplex, 2 -simplex). The set of 6 -simplices of triangulation of $\sigma_{1} \times \sigma_{2} \times \sigma_{3}$ is $\left\{\left(\left(\sigma_{1} I_{1}\right.\right.\right.$, $\left.\left.\left.\sigma_{2} \bar{I}_{1}\right) I_{2}, \sigma_{3} \bar{I}_{2}\right)\right\}$ where :

$-I_{1} \in\{012,013,023,123\}, \bar{I}_{1} \in\{3,2,1,0\}$,

$-I_{2} \in\{01,02,03,04,05,12,13,14,15,23,24,25,34,35,45\}$,

$-\bar{I}_{2} \in\{2345,1345,1245,1235,1234,0345,0245,0235,0234,0145,0135,0134$, $0125,0124,0123\}$,

\subsection{Morphism $\tau$ Between Simploidal Chain Groups and Simplicial Chain Groups}

In this section, we define a morphism $\tau$ between simploid chains and simplicial chains of the associated semi-simplicial set. We prove that $\tau$ commutes with boundary homomorphisms.

Definition of Morphism $\tau$. The simplicial chain associated to a simploid $s$ by $\tau$ is composed of simplices from $s T$ taking into account their orientation such that for any simploidal chain $c^{\square}: c^{\square} \tau \partial=c^{\square} \partial^{\square} \tau$ (cf. Fig. 4(b)). The set of $p$-simplices associated to a $p$-simploid $s$ is $s T$. To define $\tau$, we assign a sign for each simplex of $s \tau$ such that its boundary; (1) does not contain "internal" simplices. (2) has an orientation which corresponds to the orientation of simploids of the boundary of $s$.

For example on Fig. 5. semi-simplicial set $(c)$ is equivalent to simploidal set $(b)$. The boundary of $s=\sigma \times \mu$ is $a_{1}+a_{4}-a_{2}-a_{3}$. In the associated semi-simplicial set, we know that the chain corresponding to $s$ is composed of 2 -simplices $(\sigma 0, \mu 1)$ and $(\sigma 1, \mu 0)$. The unique chain composed of these two simplices that does not contain the internal edge $a_{5}$ in its boundary and such that its boundary corresponds to the boundary of $s$ is $(\sigma 1, \mu 0)-(\sigma 0, \mu 1)$.

More generally, for a $p$-simploid $s$ corresponding to the product of two simplices $\sigma_{1}$ and $\sigma_{2}$, we know that internal $(p-1)$-simplices of $s \mathfrak{T}$ are in the

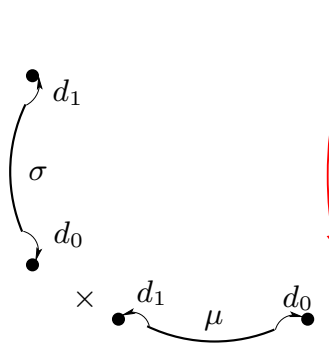

(a)

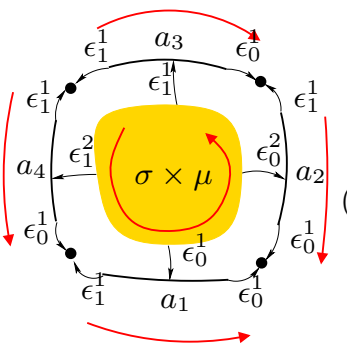

(b)

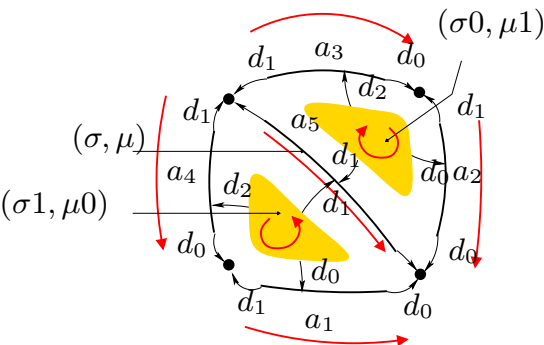

(c)

Fig. 5. (a) two simploidal sets. (b) simploidal cartesian product of $(a)$. (c) simplicial cartesian product of $(a)$. 
boundary of two simplices $\left(\sigma_{1} I, \sigma_{2} \bar{I}\right)$ and $\left(\sigma_{1} I^{\prime}, \sigma_{2} \overline{I^{\prime}}\right)$ such that there exists $i \in I, i+1 \in \bar{I}$. $\left(I^{\prime}, \overline{I^{\prime}}\right)$ is obtained from $(I, \bar{I})$ by switching $i$ and $i+1$ (cf. 9]). Thus, we have: $\left(\sigma_{1} I, \sigma_{2} \bar{I}\right) d_{i+1}=\left(\sigma_{1} I^{\prime}, \sigma_{2} \overline{I^{\prime}}\right) d_{i+1}$. For example, on Fig. 5 , the two 2-simplexes of $(b),(\sigma 0, \mu 1)$ and $(\sigma 1, \mu 0)$ have the common internal face : $(\sigma 0, \mu 1) d_{1}=(\sigma 1, \mu 0) d_{1}=(\sigma, \mu)=a_{5}$.

As each internal simplex must vanish in the boundary of a chain $s \tau$, two simplices that have a common internal face must have opposite signs. Each sign can be deduced from the parity of integer sequences that defined simplices [10], and the sign of all simploids can be deduced from simplex,

$$
\left(\left(\cdots\left(\left(\sigma_{1} \bar{J}_{a_{1}}, \sigma_{2} J_{a_{1}}\right) \bar{J}_{a_{1}+a_{2}}, \sigma_{3} J_{a_{1}+a_{2}}\right) \cdots\right) \bar{J}_{a_{1}+\cdots+a_{k-1}}, \sigma_{k} J_{a_{1}+\cdots+a_{k-1}}\right)
$$

where $J_{m}=0 \cdots m-1$. So we get the following definition :

Definition 5. Let $s=\sigma_{1} \times \cdots \times \sigma_{k}$ a simploid of length $k$ and dimension $a_{1}+\cdots+a_{k}$.

$$
s \tau=\sum_{\bar{I}_{1} \cdots \bar{I}_{k-1}}(-1)^{A\left(\bar{I}_{1}, \cdots, \bar{I}_{k-1}\right)}\left(\left(\cdots\left(\left(\sigma_{1} I_{1}, \sigma_{2} \bar{I}_{1}\right) I_{2}, \sigma_{3} \bar{I}_{2}\right) \cdots\right) I_{k-1}, \sigma_{k} \bar{I}_{k-1}\right)
$$

where:

$\left.-A\left(\bar{I}_{1}, \cdots, \bar{I}_{k-1}\right)=p\left(J_{a_{1}}\right)+p\left(\bar{I}_{1}\right)\right)+\cdots+p\left(J_{a_{1}+\cdots+a_{k-1}}\right)+p\left(\bar{I}_{k-1}\right)$

- for any integer sequence $I, p(I)$ is the parity of the sum of elements of $I$.

So we get the commutation property such that for a simploid $s, s \tau \partial=s \partial^{\square} \tau$. The proof is not provided here since it is direct and long (see [10]).

Conversion Between Simploidal and Simplicial Generators. We introduce the following notations: Let $S^{\square}$ be a simploidal set and let $S$ be its associated semi-simplicial set. $C^{\square}, Z^{\square}, B^{\square}$ et $H^{\square}$ (resp. $C, Z, B, H$ ) denote chain group, cycle group, boundary group and homology group of $S^{\square}$ (resp. $S$ ). The previous commutation property ensures that $\tau$ preserves cycles and boundaries, i.e. we use this property to prove that for any simploidal chain $c^{\square}$, if $c^{\square}$ is a cycle, then $c^{\square} \tau$ is a cycle and if $c^{\square}$ is a boundary, then $c^{\square} \tau$ is a boundary too.

Reciprocally, it can be proved that any simplicial cycle $z$ (resp. boundary) is homologous to a simplicial cycle $z^{\prime}$ (resp. boundary) such that $z^{\square} \tau=z^{\prime}$, where $z^{\square}$ is a simploidal cycle (resp. boundary). For example on Fig. 5, the simplicial chain $a_{1}-a_{5}+a_{4}$ is homologous to $a_{1}-a_{2}-a_{3}+a_{4}$ (they are both boundaries) which is the image by $\tau$ of a simploidal chain $\left(a_{1}-a_{2}-a_{3}+a_{4}\right)$.

Note that any simplicial $p$-chain $c$ can be partitioned according to their corresponding simploids, i.e. $c=\sum_{i} \sum_{j} \alpha_{i j} \sigma_{i j}$ where for a given $i$, every simplex $\sigma_{i j}$ is associated with the same simploid $s_{i}$.

Let $z=\sum_{i} \sum_{j} \alpha_{i j} \sigma_{i j}$ be a simplicial $p$-cycle (resp. boundary). We consider the following two cases: 
- Case (1): For all $i, s_{i}$ is a $p$-simploid. In this case, we can directly prove that there exists a simploidal cycle (resp. boundary) $z^{\square}=\sum_{i} \gamma_{i} s_{i}$ such that $z^{\square} \tau=z$; else $z$ contains simplices which are internal to a simploid, and $z$ is not a cycle (resp. boundary).

- Case (2): There exists $k$ such that $s_{k}$ is a $n$-simploid, $n>p$. Let $c_{k}$ be the subchain of $z$ corresponding to simploid $s_{k}$. The boundary of $c_{k}$ must be in the boundary of $s_{k}$ (since we consider only cycles). In this case, we propose an algorithm that constructs a simplicial $p$-chain $c_{k}^{\prime}$ homologous to $c_{k}$ having the same boundary, such that each simplex of $c_{k}^{\prime}$ comes from a $m$ - simploid, $m<n$. As $c_{k}^{\prime}$ is homologous and have the same boundary as $c_{k}$, by replacing $c_{k}$ by $c_{k}^{\prime}$ in the expression of $z$ we don't change the homology class of $z$. This operation is repeated until all simplices belong to $p$-simploids (corresponding to case (1)).

We do not provide here completely this algorithm as it is rather technical. The principle is to use an ordering of simplices 10 for replacing each $p$-simplex (in $s_{k} \mathfrak{T}$ ) of the current chain $c_{k}$ by its complementary in the boundary of a $(p+1)$-simplex of $s_{k} \mathfrak{T}$.

For example Fig. 6( $a$ ) represents a subchain $c_{k}$ which is a part of a 2-cycle. The two triangles of $c_{k}$ come from the triangulation of the cube, which is a 3 -simploid. (b) illustrates a chain $c_{k}^{\prime}$, homologous to $c_{k}$ and with same boundary. Each triangle of $c_{k}^{\prime}$ comes from the triangulations of simploids on the boundary of the cube.

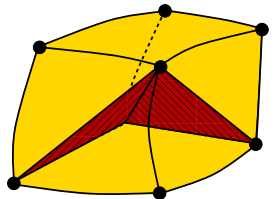

(a)

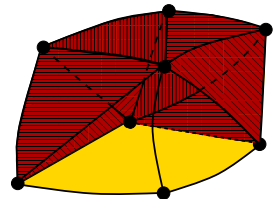

(b)

Fig. 6. (a) a 2-chain which comes from the cube (of dimension 3). (b) an homologous 2 -chain which has same boundary as. Each resulting simplex of comes from simploids of the boundary of the cube.

As a conclusion, we can associate to each simploidal cycle (resp. boundary) a simplicial cycle (resp. boundary) using $\tau$. Reciprocally, any simplicial cycle $z$ (resp. boundary) can be transformed into an homologous cycle (resp. boundary) $z^{\prime}$ such that $z^{\prime}$ is the image of a simploidal cycle (resp. boundary). So we are able to convert any generator of a simploidal set into a generator on the associated semi-simplicial set and conversely. Incidentally, this provide a purely combinatorial and constructive proof of the equivalence between simploidal and simplicial homology.

$\overline{10}$ This ordering is based on the properties of integer sequences that define simplices on a semi-simplicial set associated to a simploidal set. 


\section{Conclusion}

In this paper, notions of chains, cycles and boundaries have been extended to simploids. We defined boundary homomorphisms $\partial^{\square}$ for simploidal sets (these homomorphisms are directly defined using boundary operators $\epsilon_{j}^{i}$ ): we also proved that $c \partial^{\square} \partial^{\square}=0$ for any simploidal chain $c$. So we show how to associate a free chain complex to a simploidal set and thus we defined an homology for simploidal sets.

We provided algorithms for converting a simploidal set into an equivalent semi-simplicial set. Then we provided algorithms for converting simploidal homology generators into simplicial ones and reciprocally. We thus provide a purely combinatorial and constructive proof of the equivalence between simplicial and simploidal homology.

Now we want to study the adaptation to simploidal sets of existing algorithms initially defined for computing homology of simplicial structures [7. We hope that this will lead to interesting results in terms of memory occupation and efficiency. To do so, we need to develop comparisons with, as far as the authors know, the lone studies for non simplicial complexes [6].

From a practical point of view, we are also interested to experiment these algorithms for images of dimension greater than or equal to 3 (voxel images, sequences of $3 \mathrm{D}$ images).

\section{References}

1. Dahmen, W., Micchelli, C.A.: On the linear independence of multivariate b-splines I. Triangulation of simploids. SIAM J. Numer. Anal. 19 (1982)

2. Moore, D.: V.10 Understanding simploids. In: Graphic Gems III. Academic Press (1992) 250-255

3. Allili, M., Corriveau, D., Ziou, D.: Morse homology desriptor for shape characterization. In: Proc. ICPR 2004. (2004)

4. Munkres, J.R.: Elements of algebraic topology. Perseus Books (1984)

5. Lang, V., Lienhardt, P.: Geometric modeling with simplicial sets. In: Proc. of Pacific Graphics'95, Seoul, Korea (1995)

6. Kaczynski, T., Mischaikow, K., Mrozek, M.: Computational Homology. Springer (2004)

7. Peltier, S., Alayrangues, S., Fuchs, L., Lachaud, J.O.: Computation of homology groups and generators. In: Proceedings of 12th International Conference of Discrete Geometry for Computer Imagery. Volume 3429. (2005) 195-205

8. Fuchs, L., Lienhardt, P.: Topological structures and free-form spaces. In: Journes franco-espagnoles de gomtrie algorithmique. (1997) 35-46

9. Lienhardt, P., Skapin, X., Bergey, A.: Cartesian product of simplicial and cellular structures. Int. Journal of Computational Geometry and Applications 14(3) (2004) $115-159$

10. Peltier, S.: Calcul de groupes d'homologie sur des structures simpliciales, simploidales et cellulaires. PhD thesis, Université de Poitiers (2006) To appear. 